\title{
Penehyclidine hydrochloride alleviates lipopolysaccharide-induced acute lung injury in rats: Potential role of caveolin-1 expression upregulation
}

\author{
XIAOJING WU ${ }^{1 *}$, QIAN KONG $^{1 *}$, ZHONGYUAN XIA $^{1}$, LIYING ZHAN $^{1}$, WEINA DUAN $^{1}$ and XUEMIN SONG ${ }^{2}$ \\ ${ }^{1}$ Department of Anesthesiology, Renmin Hospital of Wuhan University, Wuhan, Hubei 430060; \\ ${ }^{2}$ Department of Anesthesiology and Critical Care Medicine, Zhongnan Hospital of \\ Wuhan University, Wuhan, Hubei 430071, P.R. China
}

Received October 25, 2018; Accepted February 26, 2019

DOI: $10.3892 /$ ijmm.2019.4117

\begin{abstract}
The aim of the present study was to examine the protective effect of caveolin-1 (Cav-1) in the penehyclidine hydrochloride (PHC)-based inhibition of lipopolysaccharide (LPS)-induced acute lung injury (ALI) in vivo and in vitro, in addition to the potential underlying mechanisms. In vivo, an ALI rat model was established via intratracheal administration of LPS $(5 \mathrm{mg} / \mathrm{kg})$, and PHC $(2 \mathrm{mg} / \mathrm{kg})$ was administered 30 min following LPS treatment. In vitro, the Cav-1 gene was knocked down by small interfering (si)RNA in J774A.1 cells. Cells were incubated with LPS $(1 \mu \mathrm{g} / \mathrm{ml})$ for $2 \mathrm{~h}$, and subsequently incubated with PHC ( $2 \mu \mathrm{g} / \mathrm{ml})$ for an additional $2 \mathrm{~h}$. Lung injury was assessed by lung histology and the ratio of polymorphonuclear leukocytes (PMNs) to total cells was assessed in bronchoalveolar lavage fluid (BALF), myeloperoxidase (MPO) activity, BALF protein content and lung wet/dry (W/D) ratio. The levels of pro-inflammatory factors, including tumor necrosis factor- $\alpha$ (TNF- $\alpha$ ), interleukin (IL)-6 and IL- $1 \beta$, in the sera of rats and cell culture supernatant were determined by ELISA. The protein expression levels of Cav-1, toll-like receptor 4 (TLR4), phosphorylated (p)-p38 mitogen
\end{abstract}

Correspondence to: Professor Xuemin Song, Department of Anesthesiology and Critical Care Medicine, Zhongnan Hospital of Wuhan University, 169 Donghu Road, Wuchang, Wuhan, Hubei 430071, P.R. China

E-mail: sxmcl1018@163.com

${ }^{*}$ Contributed equally

Abbreviations: ALI, acute lung injury; PHC, penehyclidine hydrochloride; LPS, lipopolysaccharide; Cav-1, caveolin-1; NF- $\kappa \mathrm{B}$, nuclear factor kappa-light-chain-enhancer of activated B cells; MAPK, mitogen activated protein kinase; siRNA, short-interfering RNA; TLR4, Toll-like receptor 4

Key words: penehyclidine hydrochloride, caveolin-1, acute lung injury, lipopolysaccharide, nuclear factor kappa-light-chain-enhancer of activated B cells, p38 mitogen activated protein kinases activated protein kinases (p38 MAPKs) and nuclear factor kappa-light-chain-enhancer of activated B cells transcription factor p65 subunit (NF- $\mathrm{KB}$ p65) in lung tissues and J774A.1 cells were analyzed by western blot analysis. The results indicated that PHC effectively alleviated lung injury by decreasing neutrophil infiltration and protein concentration in BALF, and the lung W/D ratio and MPO activity and pro-inflammatory cytokine production induced by LPS. Furthermore, PHC significantly decreased the degrees of histopathological changes and pulmonary dysfunction. In vitro, treatment with PHC inhibited pro-inflammatory cytokine levels and MPO activity in LPS-stimulated J774A.1 cells. However, the results in the J774A.1 cells with Cav-1 gene knockdown were contrary. In addition, PHC decreased TLR4, p-p38 MAPKs and nuclear $\mathrm{NF}-\kappa \mathrm{B}$ p65 expression levels and upregulated the expression level of Cav-1, in vivo and in vitro. These data demonstrated that PHC exhibited a protective effect against LPS-induced ALI in rats and LPS-stimulated J774A.1 cells, which may be due to the inhibition of p38 MAPKs phosphorylation and TLR4/NF- $\mathrm{B}$ signaling pathway by Cav-1 upregulation.

\section{Introduction}

Acute lung injury (ALI) is clinical syndrome of acute respiratory failure, which is caused primarily by acute lung inflammation, damaging the alveolar capillary barrier (1), followed by protein-rich edema fluid accumulation in the airway, causing diffuse pulmonary interstitial edema and abnormal gas exchange (2). The injury factors have been described as follows: Sepsis, pneumonia, aspiration of gastric contents, severe trauma with shock, severe acute pancreatitis (3-5). ALI is a life-threatening disease with high morbidity and mortality rates, and to the best of our knowledge, no specific pharmacological treatment has been established (2).

Lipopolysaccharide (LPS), a key component of the cell wall in gram-negative bacteria, is a common factor that leads to ALI (6). Toll-like receptor (TLR) signaling serves a key role in the pathogenesis of ALI. Following binding with LPS, TLR4 may induce the activation of myeloid differentiation factor 88-dependent interleukin (IL)-1 receptor activated kinase 1/4, and promote mitogen activated protein kinase (MAPK) activation 
and nuclear factor kappa-light-chain-enhancer of activated $\mathrm{B}$ cells transcription factor p65 subunit $(\mathrm{NF}-\kappa \mathrm{B}$ p65) translocation to the nucleus (7). The activation of $N F-\kappa B$ has been demonstrated to regulate the expression of a series of genes, including pro-inflammatory cytokines tumor necrosis factor- $\alpha$ (TNF- $\alpha$ ), IL-1 $\beta$ and IL-6 (8), which may lead to lung tissue damage (9). p38 MAPKs are involved in the occurrence and development of ALI caused by various etiologies $(10,11)$. Inhibition of p38 MAPKs may block the activation of $\mathrm{NF}-\kappa \mathrm{B}$, and effectively inhibit lung injury induced by LPS, including neutrophil recruitment in the lung, protein exudation and apoptosis in bronchoalveolar lavage fluid (BALF) (12). Therefore, inhibiting the activity of the TLR4/NF- $\kappa$ B and $\mathrm{p} 38$ MAPKs signaling pathways has potential therapeutic value in LPS-induced ALI.

Penehyclidine hydrochloride (PHC) is a novel anticholinergic drug, which may selectively inhibit muscarinic acetylcholine receptor M1 (M1), muscarinic acetylcholine receptor (M3) and nicotinic acetylcholine receptors N1 and $\mathrm{N} 2$ (N1 and N2), with almost no cardiovascular side effects associated with muscarinic acetylcholine receptor M2 (M2) receptors (13). It has been demonstrated that $\mathrm{PHC}$ has a protective effect in a rat model of LPS-induced ALI, and the effect may involve the inhibition of the p38 MAPKs signaling pathway and NF- $\kappa \mathrm{B}$ activation (14). However, the underlying mechanism of PHC inhibition in the activation of these signaling pathways remains unclear.

Caveolin-1 (Cav-1) is the principal structural component of caveolae, which are omega-shaped invaginations of plasma membrane (15-17). Previous studies have suggested that Cav-1 serves a crucial role in the pathogenesis of ALI, which may regulate acute inflammation and capillary leakage during lung injury (18). Wang et al (19) observed that Cav-1 in murine peritoneal and alveolar macrophages had the ability to regulate LPS-induced pro-inflammatory cytokine TNF- $\alpha$ and IL- 6 production. This effect may be due to the fact that Cav-1 inhibits LPS-induced p38 MAPKs phosphorylation and $\mathrm{NF}-\kappa \mathrm{B}$ signaling pathway activation (20).

The aim of the present study was to observe the involvement of Cav-1 in the PHC-based inhibition of LPS-induced ALI in vivo and in vitro and the potential underlying mechanisms.

\section{Materials and methods}

Reagents. LPS (E coli 0111:B4) was obtained from Sigma-Aldrich; Merck KGaA (Darmstadt, Germany). ELISA kits were purchased from R\&D Systems, Inc. (Minneapolis, MN, USA), mouse TNF- $\alpha$ (PMTA00B), IL-6 (PM6000B) and IL-1 $\beta$ (PMLB00C) Quantikine ELISA kit, rat TNF- $\alpha$ (PRTA00), IL-6 (PR6000B) and IL-1 $\beta$ (PRLB00) Quantikine ELISA kit. The myeloperoxidase (MPO) kit was obtained from Nanjing Jiancheng Bioengineering Institute (Nanjing, China). PHC was purchased from Chengdu List Pharmaceutical Co. Ltd. (Chengdu, China).

Animal model. Male Sprague-Dawley (SD) rats (170-190 g; 8-10 weeks-old) were purchased from Beijing Vital River Laboratory Animal Technology Co., Ltd (Beijing, China; certificate no. SCXK2016-0006). The rats were housed in a specific pathogen-free, laminar-flow atmosphere under controlled temperature $\left(25 \pm 2^{\circ} \mathrm{C}\right)$, humidity $(50 \pm 10 \%)$, and $12 \mathrm{~h}$ light: Dark cycle, with ad libitum access to food and water. The animals acclimated to this environment for 1 week prior to the experiment. The present study was approved by Medical Ethics Committee of Renmin Hospital of Wuhan University and was performed in accordance with the National Institutes of Health Guidelines for the Care and Use of Laboratory Animals (21).

LPS-induced ALI in rats. Male SD rats were randomly divided into 3 groups: i) The control group (C group); ii) ALI model group (LPS group); and iii) ALI + PHC treatment group (P + LPS group). The ALI model was established using a protocol based on the study by Shen et al (14), by intratracheal instillation of LPS. A total of $0.2 \mathrm{ml} \mathrm{LPS}(5 \mathrm{mg} / \mathrm{kg})$ was administered to rats in the LPS and P + LPS groups by intratracheal instillation, while rats in the $\mathrm{C}$ group received an equal volume of normal saline. A total of $30 \mathrm{~min}$ later, the rats in the P + LPS group received an intraperitoneal (i.p.) injection

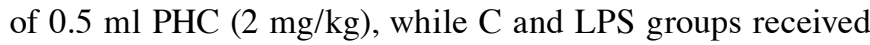
$0.5 \mathrm{ml}$ normal saline. The doses of PHC were selected based on data from our previous studies $(22,23)$. At $24 \mathrm{~h}$ after LPS treatment, the rats were anesthetized by an i.p. injection of pentobarbital (50 mg/kg; Sigma-Aldrich; Merck KGaA), and arterial blood, BALF and lung tissue samples were collected. Animal death was confirmed by observation of apnea and asystole. Lung tissues were snap-frozen in liquid nitrogen, and stored at $-80^{\circ} \mathrm{C}$ for subsequent analysis.

Arterial blood gas analysis. Following anesthesia, arterial blood samples were collected from the rats with a heparinized syringe from the carotid artery, followed by thoracotomy and alveolar lavage and removal of lung tissues. The arterial blood samples were immediately injected into an ABL700 Radiometer (Radiometer America Inc, Brea, CA, USA) to measure $\mathrm{pH}$ value, partial gas pressures of oxygen $\left(\mathrm{PaO}_{2}\right)$ and carbon dioxide $\left(\mathrm{PaCO}_{2}\right)$, and lactic acid (Lac).

Histopathological lung examination. The right lung lobes were excised, washed and fixed in $4 \%(\mathrm{v} / \mathrm{v})$ paraformaldehyde $\left(4^{\circ} \mathrm{C}, 12 \mathrm{~h}\right)$. Lung tissues were embedded in paraffin, sectioned at $4 \mu \mathrm{m}$ thickness, and stained with hematoxylin and eosin (H\&E) solution (Sigma-Aldrich; Merck KGaA) (0.2\% hematoxylin staining for $10 \mathrm{~min}$, and $0.5 \%$ eosin staining for $2 \mathrm{~min}$; each at room temperature) to estimate inflammation in the peribronchial and alveolar lesions. The stained slides were then observed with a light microscope (BX51; Olympus Corporation, Tokyo, Japan; magnification, x100) and the digital micrographs were captured for analysis. The histologic injury scores were evaluated from 0 to 4 , as described previously (24). Briefly, the degree of lung injury was graded according to the following scoring system: 0 , no injury and appears normal; 1 , minimal (injury up to $25 \%$ of the field); 2 , mild (injury between $25-50 \%$ of the field); 3 , moderate (injury between $50-75 \%$ of the field); and 4 , severe ( $>75 \%$, diffuse injury). Tissue sections were examined by a pathologist blinded to the experiment.

Ultrastructural changes of lung tissues under transmission electron microscopy. The lungs were isolated and cut into 1-2 $\mathrm{mm}^{3}$ cubes. Lung tissue samples were fixed by immersion 
in a $2.5 \%$ buffer of glutaraldehyde for $24 \mathrm{~h}$ at $4^{\circ} \mathrm{C}$, washed by PBS solution 3 times, post-fixed for $1 \mathrm{~h}$ at room temperature in $1 \%$ osmium tetroxide, dehydrated in graded concentrations of ethyl alcohol (30, 50, 70, 90 and 100\%), and embedded in epoxy resin. Ultrathin sections $(70 \mathrm{~nm})$ were double-stained with $1 \%$ uranyl acetate for $30 \mathrm{~min}$ at room temperature and lead citrate for $15 \mathrm{~min}$ at room temperature. Subsequently the sections were examined under a transmission electron microscope (JEOL 100 CX; JEOL, Ltd., Tokyo, Japan).

BALF collection and inflammatory cell counting. To obtain the BALF, ice-cold PBS $(2 \mathrm{ml})$ was infused into the lungs of the rats 3 times and withdrawn each time using a tracheal cannula (a total volume of $6 \mathrm{ml}$ ). The collected BALF was then centrifuged at $200 \mathrm{x}$ g for $15 \mathrm{~min}$ at $4^{\circ} \mathrm{C}$. Following centrifugation, the supernatant was stored at $-80^{\circ} \mathrm{C}$ for subsequent assays. Following exclusion of dead cells by trypan blue staining $(0.5 \mathrm{ml} 0.4 \%$ trypan blue solution was transferred to the test tube. Subsequently, $0.3 \mathrm{ml}$ Hanks balanced salt solution (HBSS) and $0.2 \mathrm{ml}$ cell suspension were added and mixed sufficiently and allowed to stand for 5 to $15 \mathrm{~min}$. All the procedures were performed at room temperature. The stained cell suspension was absorbed and transferred to the blood cell count board, and the number of living and dead cells were then counted under a light microscope (BX51; Olympus, Tokyo, Japan; magnification, $\mathrm{x} 400$ ). The total inflammatory cells in BALF were determined by counting cells with a hemocytometer (cat. no. 3100; Hausser Scientific, Horsham, PA, USA). In order to analyze differential cell counting, $100 \mu \mathrm{l}$ BALF was centrifuged $\left(200 \mathrm{x} \mathrm{g}\right.$ for $10 \mathrm{~min}$ at $\left.4^{\circ} \mathrm{C}\right)$ onto slides using a Cytospin (Thermo Fisher Scientific, Inc., Waltham, MA, USA). Subsequent to natural drying of the slides, the cells were fixed, and stained using Wright's Stain solution (Sigma-Aldrich; Merck KGaA) according to the manufacturer's protocol. Several drops of Wright's Stain were added to cover the slides followed by incubation for approximately $1 \mathrm{~min}$ at room temperature. Then we added the same amount of phosphate buffer ( $\mathrm{pH} 6.4$ ) and shaked the slides gently, and allowed to stand for $5 \mathrm{~min}$. The slides were then rinsed gently with ultrapure water 3 times. After the slides were dried naturally, they were examined under a light microscope (magnification, $\mathrm{x} 1,000$; oil immersion lens). The number of polymorphonuclear neutrophils (PMNs) was classified to obtain the percentage of neutrophils.

Protein content determination in BALF. The frozen BALF supernatant was thawed and thoroughly mixed. The total protein concentration was determined using a bicinchoninic acid method.

Lung wet/dry (W/D) weight ratio. The water content of the lungs was determined by calculating the W/D weight ratio of the lung tissues. The left lobe of the lung was excised, washed with PBS, blotted and then weighed to obtain the 'wet' weight. The lung was then dried at $65^{\circ} \mathrm{C}$ for $48 \mathrm{~h}$ to obtain the 'dry' weight. The W/D ratio was calculated to assess the degree of pulmonary edema.

MPO activity assay. Radioimmunoprecipitation assay lysis buffer (Sigma-Aldrich; Merck KGaA) was used for lysing tissues, and $10 \mathrm{mg}$ tissue was obtained for each sample.
Following washing with cold PBS, the tissues were resuspended in 4 volumes of MPO Assay buffer (included with the Myeloperoxidase Activity Assay kit), and then centrifuged at $4^{\circ} \mathrm{C}$ and $13,000 \mathrm{x}$ g for $10 \mathrm{~min}$. The supernatant was collected and transferred to a clean tube, which was then placed on ice. The MPO activity was assayed using Myeloperoxidase Activity Assay kit (cat. no. ab105136; Abcam, Cambridge, MA, USA), by measuring the absorbance of the sample at $460 \mathrm{~nm}$ using a microplate reader (Bio-Rad Laboratories, Inc., Hercules, CA, USA). The specific MPO activity in the lungs was measured as units/mg protein.

Measurement of pro-inflammatory cytokines in serum. Blood was collected from the carotid artery, and the serum was obtained following centrifugation at $1,000 \mathrm{x} \mathrm{g}$ for $10 \mathrm{~min}$ at $4^{\circ} \mathrm{C}$ and used for ELISA analysis. The levels of TNF- $\alpha$, IL- 6 and IL- $1 \beta$ in serum were determined using ELISA kits according to the manufacturer's instructions (R\&D Systems, Inc., Minneapolis, MN, USA). The absorbance was measured at $450 \mathrm{~nm}$ using an ELISA reader (BioTek Instruments, Inc., Winooski, VT, USA).

Western blot analysis. A total of $24 \mathrm{~h}$ after LPS injection, the lung tissues were harvested and snap-frozen in liquid nitrogen until homogenization. The lung tissues were homogenized using a homogenizer with tissue nuclear and cytoplasmic protein extraction reagents (Sigma-Aldrich; Merck KGaA), according to the manufacturer's protocol. Protein concentrations were determined using a BCA protein assay kit. Equal amounts of protein $(40 \mu \mathrm{g})$ were loaded per well on $12 \%$ SDS-PAGE gels and transferred onto polyvinylidene difluoride membranes. The resulting membranes were blocked by incubation with $5 \%$ skim milk in TBS + Tween-20 (TBST) $(0.1 \%$ Tween-20) at room temperature for $2 \mathrm{~h}$ on a rotary shaker, followed by washing with TBST. Subsequently, the membranes were incubated with specific primary antibodies at $4^{\circ} \mathrm{C}$ overnight: Rabbit anti-TLR4 (1:300; cat. no. ab217274; Abcam, Cambridge, UK Abcam, Cambridge, UK); Cav-1 (1.2 $\mu \mathrm{g} / \mathrm{ml}$; cat. no. ab2910); p38 MAPKs (1.8 $\mu \mathrm{g} / \mathrm{ml}$; cat. no. ab27986); phosphorylated (p)-p38 MAPKs (1:600; cat. no. ab47363); NF-кB p65 (0.5 $\mu \mathrm{g} / \mathrm{ml}$; cat. no. ab16502; all Abcam); GAPDH (1:1,000; cat. no. 5174; Cell Signaling Technology, Inc., Danvers, MA, USA); and histone H3 antibody (1:2,000; cat. no. 4499; Cell Signaling Technology, Inc., Danvers, MA, USA). The membranes were then washed with TBST followed by incubation with the horseradish peroxidase (HRP)-conjugated secondary antibody (1:2,000; cat. no. sc-2004; Santa Cruz Biotechnology, Inc., Dallas, TX, USA) at room temperature for $1 \mathrm{~h}$. The blots were washed with TBST and detected using an enhanced chemiluminescence (ECL) western blotting detection kit (32132; Thermo Fisher Scientific, Inc. Waltham, MA, USA). The proteins bands were then observed using an ECL western blotting analysis system (Bio-Rad Laboratories, Inc.) and quantified by densitometry using Image Lab v5.2.1 software (Informer Technologies, Inc.).

J774A.1 cells culture and treatment. The mouse monocyte/macrophage J774A.1 cell line was purchased from the American Type Culture Collection (Rockville, MD, USA). The J774A.1 cells were seeded (desity, $2 \times 10^{5} / \mathrm{ml}$ ) onto a 
$6 \mathrm{~cm}$ dish and were cultured in Dulbecco's modified Eagle's medium (DMEM; 11965092) supplemented with $10 \%$ fetal bovine serum (26400044; both Thermo Fisher Scientific, Inc.), $50 \mathrm{U} / \mathrm{ml}$ penicillin $\mathrm{G}$ and $50 \mu \mathrm{g} / \mathrm{ml}$ streptomycin, and maintained at $37^{\circ} \mathrm{C}$ in a humidified atmosphere containing $5 \% \mathrm{CO}_{2}$. The cells were grown until 50-70\% confluence prior to treatment. The cells were divided into 5 groups, as follows: i) The scramble (Scr)-short interfering RNA (siRNA) control (S group); ii) the Scr-siRNA + LPS (LPS group); iii) the Scr-siRNA + PHC + LPS (P + LPS group); iv) the SMARTpool Cav-1-siRNA + LPS (C + LPS group); and the SMARTpool Cav-1- siRNA + PHC + LPS (C + P + LPS group). According to the manufacturer's protocol, Scr-siRNA (D-001810-10-20) and SMARTpool Cav-1-siRNAs (12389) were added to the cells (50-70\% confluence) using DharmaFECT Transfection Reagent (T-2001-03; all Dharmacon, Lafayette, CO, USA). The siRNA sequences against CAV-1 (CAV1-1 siRNA) were 5'-AGACGAGCUGAGCGAGAAGCA-3' (sense) and 5'-UGC UUCUCGCUCAGCUCGUCU-3' (antisense) and (CAV1-2 siRNA) 5'-CAU-CUACAAGCCCAACAAC-3' (sense) and 5'-GUUGUUGGGCUUGUAGAUG-3' (antisense). The final siRNA concentrations were $50 \mathrm{nM}$. Cells in the $\mathrm{S}$, LPS and P + LPS groups were transfected with Scr-siRNA for $48 \mathrm{~h}$, and cells in the C + LPS and C + P + LPS groups were transfected with SMARTpool Cav-1-siRNAs for $48 \mathrm{~h}$. Following transfection, the cells in all groups, with the exception of the $\mathrm{S}$ group, were incubated in the presence of LPS $(1 \mu \mathrm{g} / \mathrm{ml})$ at $37^{\circ} \mathrm{C}$ for $2 \mathrm{~h}$, and then cells in the $\mathrm{P}+\mathrm{LPS}$ and $\mathrm{C}+\mathrm{P}+$ LPS groups were incubated with PHC $(2 \mu \mathrm{g} / \mathrm{ml})$ at $37^{\circ} \mathrm{C}$ for an additional $2 \mathrm{~h}$. The protein expression levels of Cav-1, TLR4, p38 MAPKs, p-p38 MAPKs and nuclear NF- $\mathrm{B}$ p65 were the determined by western blot analysis, as described in the above paragraph. The primary antibodies used were: Rabbit anti-TLR4 (1:500; cat. no. ab13556); Cav-1 (1.5 $\mu \mathrm{g} / \mathrm{ml}$; cat. no. ab2910); p38 MAPKs (1.5 $\mu \mathrm{g} / \mathrm{ml}$; cat. no. ab27986; all Abcam); p-p38 MAPKs (1:800; cat. no. ab47363); NF-кB p65 $(0.5 \mu \mathrm{g} / \mathrm{ml}$; cat. no. ab16502; both Abcam, Cambridge, UK); GAPDH (1:1,000; cat. no. 5174); and histone H3 antibody (1:2,000; cat. no. 4499; both Cell Signaling Technology, Inc.). HRP-conjugated secondary antibody (1:2,000; cat. no. sc-2004; Santa Cruz Biotechnology, Inc.) was selected as the secondary antibody. The levels of TNF- $\alpha$, IL- 6 and IL- $1 \beta$ in cell culture supernatant were determined using Quantikine ELISA kits. The MPO activity was measured by colorimetry, as mentioned above.

Statistical analysis. The data are expressed as the mean \pm standard error of the mean. The statistical analysis was performed using GraphPad Prism (version 7.0; GraphPad Software, Inc., La Jolla, CA, USA), and groups were compared using a one-way analysis of variance followed by Dunnett's least significant difference post-hoc test. $\mathrm{P}<0.05$ was considered to indicate a statistically significant difference.

\section{Results}

Effect of PHC on LPS-mediated lung histopathological changes. To evaluate the histological changes of the lung tissues following LPS treatment, tissues were harvested at $24 \mathrm{~h}$ following LPS stimulation and subjected to $\mathrm{H} \& \mathrm{E}$ staining. No histological changes were observed in the lung tissues of rats in the $\mathrm{C}$ group (Fig. 1A). Significant pathological changes were observed in lung tissues of LPS-treated rats, including pulmonary capillary congestion, pulmonary interstitial edema, mass inflammatory cell infiltration and alveolar wall thickening (Fig. 1B). Treatment with PHC significantly decreased the histopathological changes induced by LPS (Fig. 1C). In addition, as indicated in Fig. 1D, quantitative scoring of histological lung injury in the ALI rats was increased compared with the $\mathrm{C}$ group at $24 \mathrm{~h}$ following LPS administration. However, PHC pre-treatment decreased the pathological scores of the lung tissues.

Effect of PHC on LPS-mediated lung ultrastructural changes. Transmission electron microscopy was used to examine the ultrastructural changes in lung tissues. Lung tissues from the control group indicated normal structure (Fig. 2A). Lung tissues from LPS-treated rats exhibited enlarged mitochondria and disrupted mitochondrial cristae in the alveolar epithelial cells, lamellar corpuscle vacuole-like degeneration and degranulated rough endoplasmic reticulum membrane (Fig. 2B). In the $\mathrm{P}+$ LPS group, which received PHC pre-treatment, the level of pathological damage was significantly decreased compared with the LPS group (Fig. 2C).

Effect of PHC on pulmonary inflammation and vascular permeability during LPS-induced ALI. To examine the effects of PHC on LPS-induced pulmonary inflammation and vascular permeability, the ratio of PMNs to the total number of cells in the BALF, MPO activity, BALF protein content and lung W/D ratio were analyzed at $24 \mathrm{~h}$ following LPS injection. As indicated in Fig. 3, following LPS stimulation, the ratio of PMNs to total cells in the BALF (Fig. 3A), MPO activity (Fig. 3B), BALF protein content (Fig. 3C) and lung W/D ratio (Fig. 3D) were significantly increased compared with the $\mathrm{C}$ group. However, the increase in lung injury index was decreased by pre-treatment with $\mathrm{PHC}$.

Effects of PHC on the arterial blood gas of rats with LPS-induced lung injury. As presented in Fig. 4, compared with the control group, the arterial blood gas analysis of rats receiving LPS treatment indicated a significant change, as pH (Fig. 4A) and $\mathrm{PaO}_{2}$ (Fig. 4B) decreased, and the levels of $\mathrm{PaCO}_{2}$ (Fig. 4C) and Lac (Fig. 4D) increased. Compared with the LPS group, the $\mathrm{pH}$ and $\mathrm{PaO}_{2}$ were increased in the $\mathrm{P}+\mathrm{LPS}$ group, while the levels of $\mathrm{PaCO}_{2}$ and Lac were decreased.

Effect of PHC on pro-inflammatory cytokine production in the sera isolated from rats with LPS-induced ALI. TNF- $\alpha$, IL-6 and IL-1 $\beta$ expression levels in the sera were significantly increased in LPS-treated rats compared with the control group. By contrast, PHC-treated rats exhibited a significant decrease in TNF- $\alpha$, IL- 6 and IL-1 $\beta$ expression levels compared with the LPS group (Fig. 5).

Effect of PHC on protein expression in the lung tissue of rats with LPS-induced ALI. Rats with LPS-induced ALI exhibited increased TLR4, p-p38 MAPKs and nuclear NF- $\kappa$ B p65 expression levels, and decreased Cav-1 expression levels in the lung tissue samples compared with the control group. However, 



Figure 1. Histological assessment of the effect of PHC on LPS-induced acute lung injury. Representative histopathological images of lung tissues sections under a light microscope at magnification, $\mathrm{x} 100$ in the (A) C group (B) LPS group and (C) P + LPS group. (D) Lung injury score. The data are presented as the mean \pm standard error of the mean $(\mathrm{n}=10) .{ }^{*} \mathrm{P}<0.05$ vs. C group. ${ }^{~} \mathrm{P}<0.05$ vs. LPS group. PHC/P, penehyclidine hydrochloride; LPS, lipopolysaccharide; C, control.

A

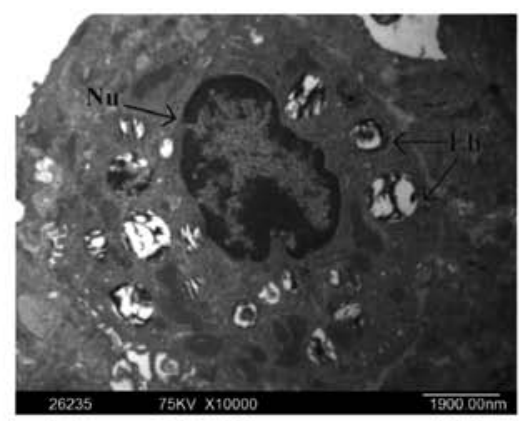

$\mathrm{B}$

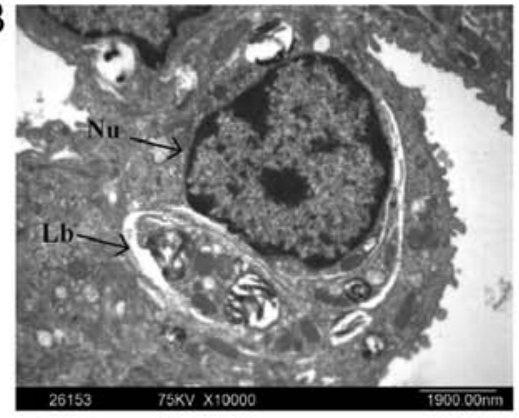

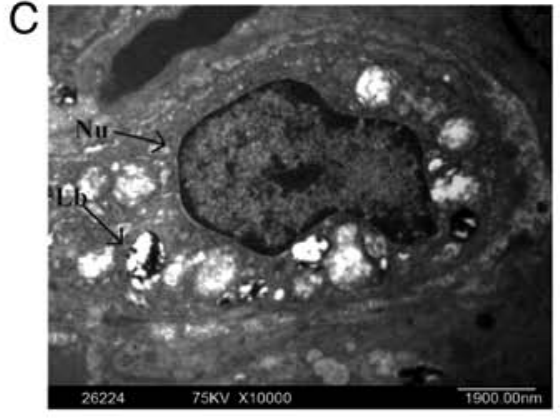

Figure 2. Ultrastructural changes in the lung tissues of rats at magnification, $\mathrm{x} 10,000$. The lung tissues were harvested at $24 \mathrm{~h}$ following LPS stimulation, and transmission electron microscopy was used to examine the ultrastructural changes in (A) C group, (B) LPS group and (C) P+LPS group. Lb, lamellar body; $\mathrm{Nu}$, nucleus; LPS, lipopolysaccharide; P, penehyclidine hydrochloride; C, control.

there was a significant decrease in TLR4, p-p38 MAPKs and nuclear NF- $\kappa \mathrm{B}$ p65 expression levels, and an increase in Cav-1 expression levels in the lung tissue of PHC-treated rats compared with the LPS-treated rats. There was no statistically significant difference in $\mathrm{p} 38$ MAPKs protein expression among the different groups (Fig. 6).

Effect of PHC on pro-inflammatory cytokine production in cell culture supernatant in LPS-stimulated J774A.1 cells. Supernatants were collected from the LPS/PHC treated J744.A1 cells in the presence of Scr-siRNA or SMARTpool Cav-1-siRNA. As presented in Fig. 7, treatment with LPS resulted in a significant increase in pro-inflammatory cytokines production in supernatants compared with the negative control group. Compared with the LPS group, the expression levels of TNF- $\alpha$, IL- 6 and IL- $1 \beta$ in the cell culture supernatant were decreased in the LPS + P group; however, the C + LPS group with Cav-1 knockdown exhibited the opposite result. The expression levels of TNF- $\alpha$, IL- 6 and IL-1 $\beta$ in the supernatant in the C + LPS + P group were increased compared with the LPS + P group, however, they were decreased compared with the $\mathrm{C}+$ LPS group.

Effect of PHC on MPO activity in LPS-stimulated J774A.I cells. Compared with the negative control group, MPO activity was significantly increased in LPS-stimulated J774A.1 cells. 
A
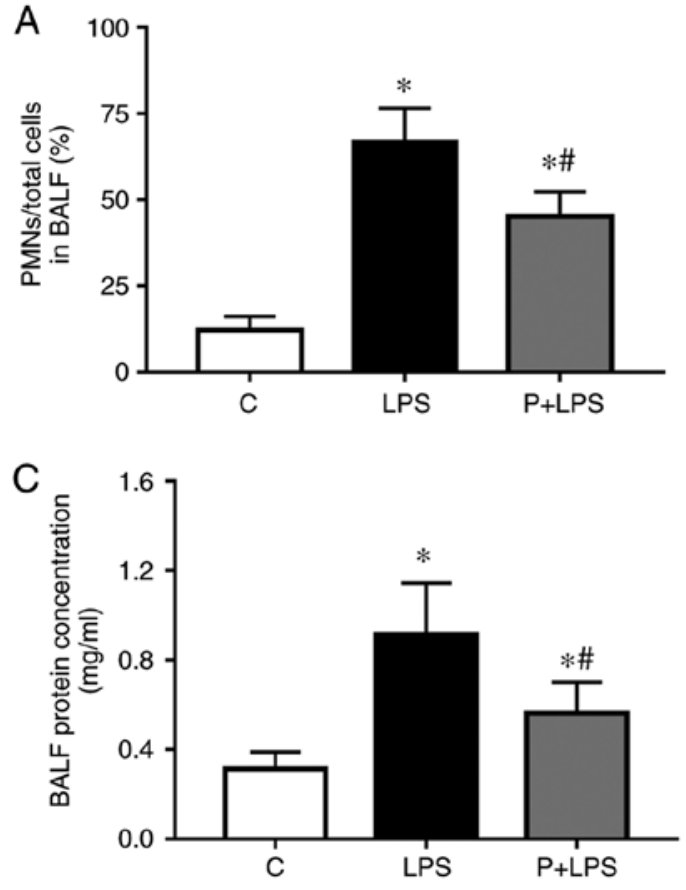

B

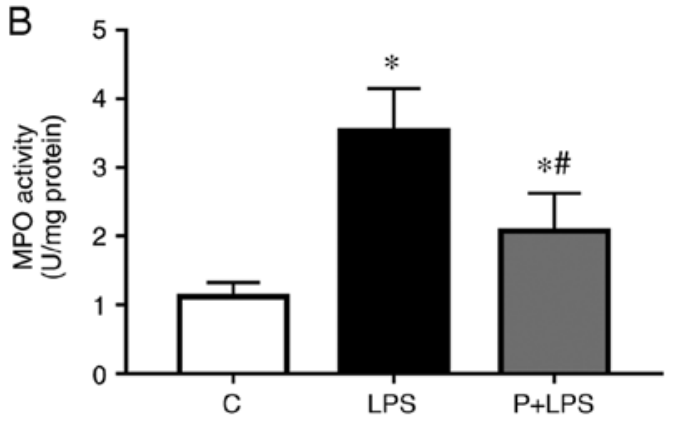

D

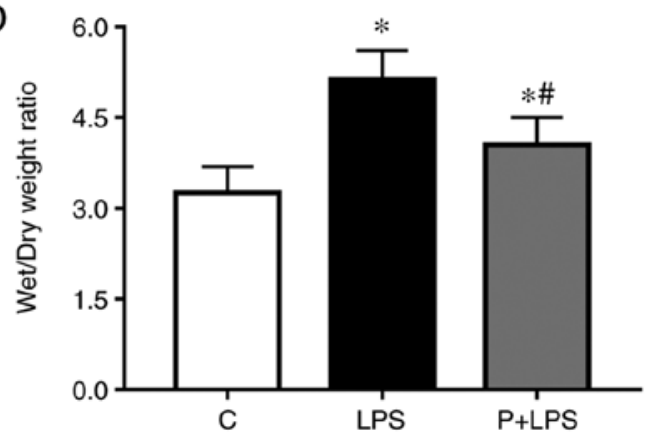

Figure 3. PHC pre-treatment significantly decreases pulmonary inflammation and vascular permeability during LPS-induced acute lung injury. (A) The ratio of PMNs to total cells in BALF and (B) MPO activity in lung tissues. (C) Total protein concentration of BALF. (D) The dry and wet weight ratio of lung tissues. The data are presented as the mean \pm standard error of the mean $(n=10)$. ${ }^{*} \mathrm{P}<0.05$ vs. C group. ${ }^{\text {P }}<0.05$ vs. LPS group. PHC/P, penehyclidine hydrochloride; LPS, lipopolysaccharide; PMNs, polymorphonuclear leukocytes; BALF, bronchoalveolar lavage fluid; MPO myeloperoxidase; C, control.
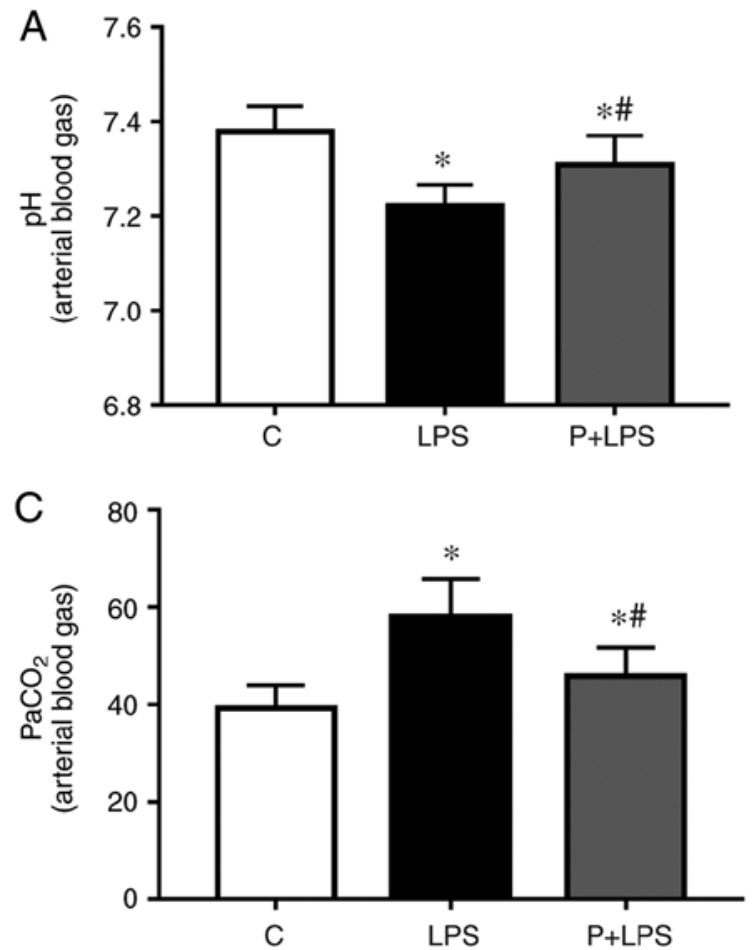
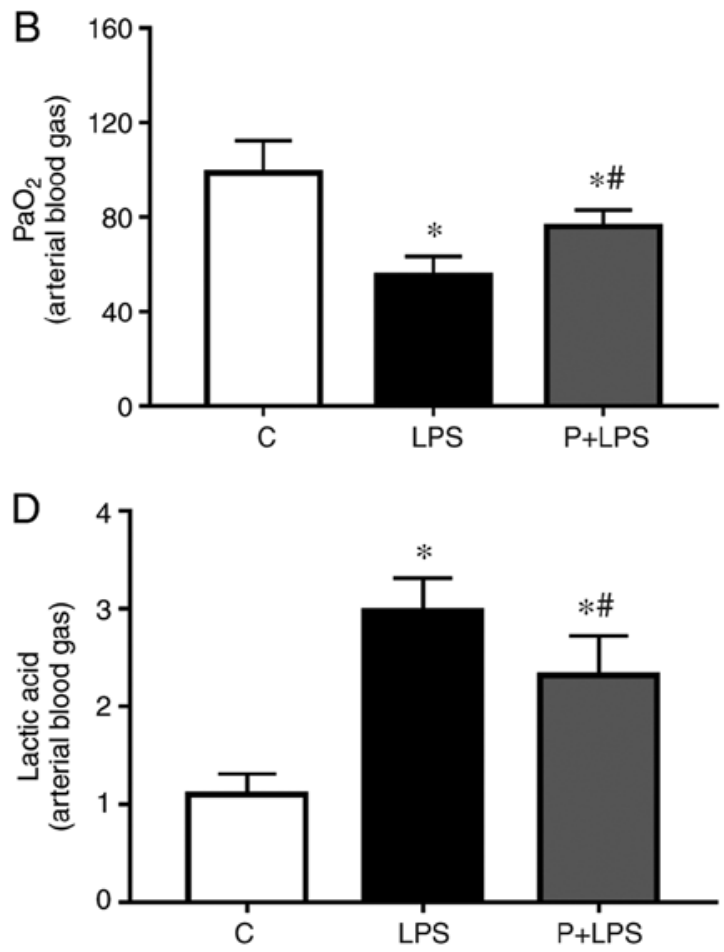

Figure 4. Effects of PHC on the arterial blood gas of rats with LPS-induced lung injury. Arterial blood sample was collected from the carotid artery $24 \mathrm{~h}$ following LPS challenge, for arterial blood gas analysis of (A) $\mathrm{pH}(\mathrm{B}) \mathrm{PaO}_{2}(\mathrm{C}) \mathrm{PaCO}_{2}$ and (D) Lac. The data are presented as the mean \pm standard error of the mean $(\mathrm{n}=10)$. ${ }^{*} \mathrm{P}<0.05$ vs. $\mathrm{C}$ group. ${ }^{\#} \mathrm{P}<0.05$ vs. LPS group. $\mathrm{PaO}_{2}$, partial pressure of arterial oxygen; $\mathrm{PaCO}_{2}$, partial pressure of arterial carbon dioxide; Lac, lactic acid; PHC/P, penehyclidine hydrochloride; LPS, lipopolysaccharide; C, control.

Compared with the LPS group, MPO activity was decreased in the LPS + P group; however, the C + LPS group with Cav-1 knockdown exhibited the opposite result. MPO activity in the group $\mathrm{C}+\mathrm{LPS}+\mathrm{P}$ was increased compared with the LPS $+\mathrm{P}$ group, however, it was decreased compared with the C + LPS group (Fig. 7D). 


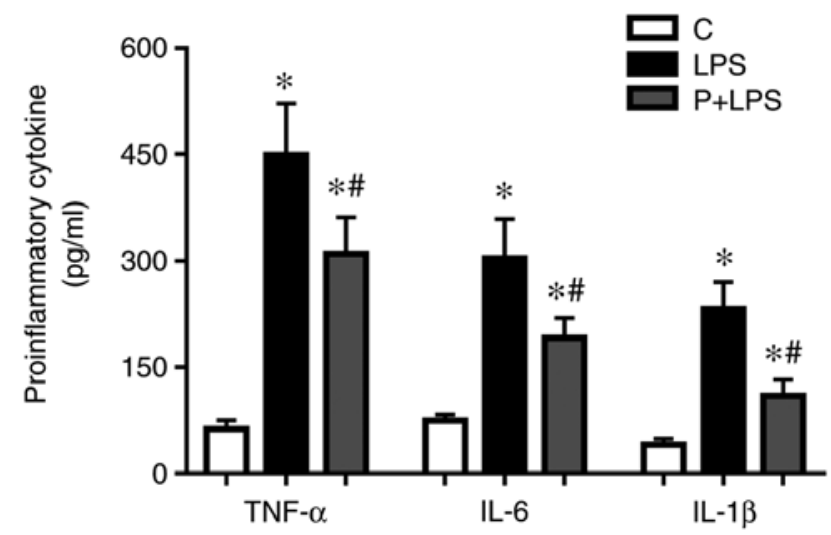

Figure 5. Effect of PHC on pro-inflammatory cytokine production in the serum isolated from rats with LPS-induced acute lung injury. The data are presented as the mean \pm standard error of the mean $(n=10)$. $\mathrm{P}<0.05$ vs. $\mathrm{C}$ group. ${ }^{\#} \mathrm{P}<0.05$ vs. LPS group. PHC/P, penehyclidine hydrochloride; LPS, lipopolysaccharide; C, control; TNF- $\alpha$, tumor necrosis factor $\alpha$; IL, interleukin.

Effect of PHC on protein expression levels in LPS-stimulated J774A.1 cells. The involvement of Cav-1 signaling in macrophages was examined directly in cross-protection experiments, in which Cav-1 expression was knocked down using siRNA. J744.A1 cells were transfected with Cav-1 siRNA and a control siRNA, which targeted a non-specific gene. The results of the western blot analysis indicated that Cav-1 siRNA transfection successfully knocked down Cav-1 in J744.A1 cells (Fig. 8A). As indicated in Fig. 8, TLR4, p-p38 MAPKs and nuclear NF- $\mathrm{B}$ p65 protein expression levels were significantly increased in LPS-stimulated J774A.1 cells, while the Cav-1 protein expression level was significantly decreased, with the exception of the LPS + P group. Compared with the LPS group, TLR4, p-p38 MAPKs and nuclear NF- $\kappa$ B p65 protein expression levels were decreased and Cav-1 protein level was increased in the LPS + P group; however, the C + LPS group with Cav-1 knockdown exhibited the opposite result. Compared with the LPS + P group, TLR4, p-p38 MAPKs and nuclear NF- $\mathrm{BB}$ p65 protein expression levels were increased, whereas Cav-1 expression protein level was decreased in the $\mathrm{C}+\mathrm{LPS}+\mathrm{P}$ group. Cells in the $\mathrm{C}+\mathrm{LPS}+\mathrm{P}$ group exhibited significantly decreased TLR4, p-p38 MAPKs and nuclear NF- $\mathrm{B}$ p65 expression levels and increased Cav-1 expression compared with the C + LPS group. There was no statistical difference in $\mathrm{p} 38 \mathrm{MAPKs}$ protein expression among the different groups.

\section{Discussion}

ALI/acute respiratory distress syndrome (ARDS) is a complex respiratory disorder associated with high morbidity and mortality rates worldwide (25). In the present study, it was suggested that Cav-1 may be involved in the protective role of PHC against LPS-induced ALI. In addition, the potential underlying mechanism associated with the inhibition of $\mathrm{p} 38$ MAPKs phosphorylation and TLR4/NF- $\kappa$ B signaling pathway activation was examined.

LPS is recognized as an important participant in the pathogenesis of sepsis (26), which may induce ALI (27). In the present study, it was indicated that intratracheal injection of LPS caused histological changes of lung tissues and dysfunction of gas exchange, indicating that ALI was successfully induced by LPS in SD rats.

$\mathrm{NF}-\kappa \mathrm{B}$ and MAPKs signal transduction pathways have been demonstrated to mediate inflammatory responses in the lung. Following recognition by TLR4, LPS causes inhibitor of $\kappa \mathrm{B}$ phosphorylation and degradation, which subsequently induces $\mathrm{NF}-\kappa \mathrm{B}$ p65 translocation to the nucleus (28). The activated $\mathrm{NF}-\kappa \mathrm{B}$ in the nucleus promotes the transcription of specific targeting genes, including the pro-inflammatory cytokines TNF- $\alpha$, IL-6 and IL-1 $\beta$ (29-31). In patients with ALI, NF- $\kappa$ B activation significantly increases pro-inflammatory cytokine production, which in turn promotes neutrophil recruitment into the lung and inflammatory responses (32). The MAPKs are a group of signaling molecules (33), among which p38 MAPKs have been demonstrated to be involved in the development of ALI/ARDS caused by different stimuli $(10,11,34)$. Following LPS challenge, p38 MAPKs are activated, which subsequently induces the release of pro-inflammatory cytokines $(35,36)$, neutrophil recruitment into the lung and bronchoconstriction (37). Inhibition of p38 MAPKs efficiently decreases the effects of LPS-induced ALI, including protein leakage and cell apoptosis in BALF, and neutrophil recruitment into the lung (12). In the present study, LPS challenge induced p38 MAPKs phosphorylation and NF- $\mathrm{B}$ p65 translocation to the nucleus in vivo and in vitro.

PHC is a novel anti-cholinergic drug, which may selectively block M1 and M3 receptors, and nicotinic acetylcholine receptors, but has almost no demonstrated cardiovascular side effects associated with $\mathrm{M} 2$ receptors. A number of studies have suggested that PHC exhibits a protective effect in pulmonary diseases and sepsis, potentially by inhibiting pro-inflammatory cytokine production (38). According to Shen et al (14), a single injection of PHC significantly decreased inflammation and lung vascular leakage in a rat model of LPS-induced ALI, as the protective effect may involve the inhibition of $\mathrm{NF}-\kappa \mathrm{B}$ and p38 MAPKs signaling pathways. In the present study indicated that PHC post-treatment in vivo decreased the degree of pulmonary edema in the rat model of LPS-induced ALI, which was additionally verified by a significant decrease in the lung W/D ratio, PMNs/total cells and total protein concentration in BALF, and was associated with decreases in histological lung damage and improvements in gas exchange dysfunction induced by LPS. In addition, PHC significantly decreased MPO activity in the lung tissues of ALI rats and LPS-stimulated J774A.1 macrophages. PHC significantly inhibited LPS-induced pro-inflammatory cytokine production in vivo and in vitro, which indicated that PHC possesses anti-inflammatory properties to inhibit the production of cytokines in LPS-induced ALI. To examine the underlying anti-inflammatory mechanism of PHC on LPS-induced ALI, the effects of PHC on NF- $\kappa \mathrm{B}$ and p38 MAPKs pathway activation were determined in vivo and in vitro. The results indicated that LPS-induced TLR4/NF- $\kappa$ B and p38 MAPKs activation were significantly inhibited by PHC administration.

Cav-1 has been demonstrated to serve a crucial role in acute inflammation and capillary leakage during ALI: Yuan et al (39) observed that $\mathrm{Cav}_{-1} \mathrm{1}^{-/}$mice exhibited an elevated lung inflammatory response, an elevated pro-inflammatory cytokine production and an increased mortality rate following LPS challenge, which may be associated with $N F-\kappa B$ activation. 

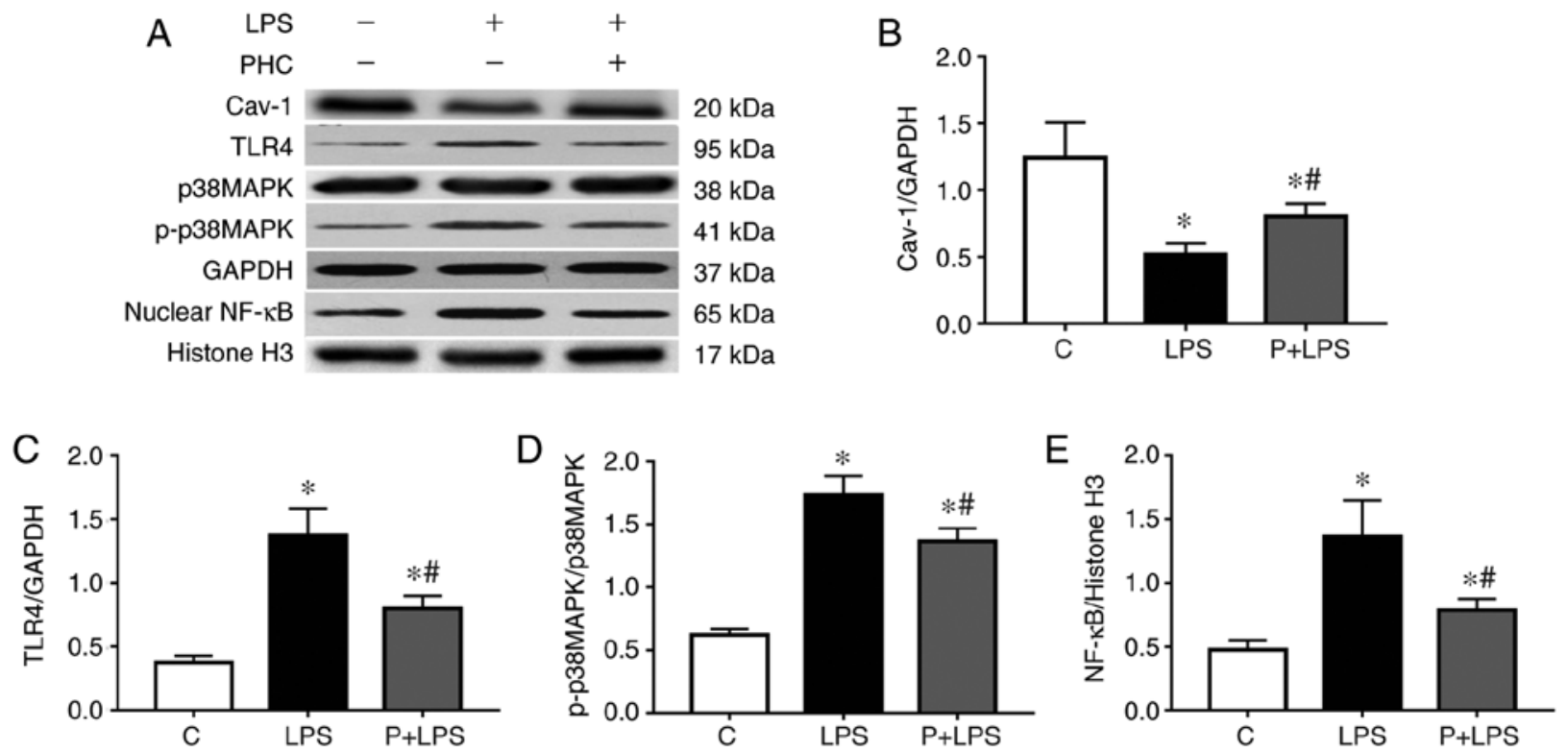

Figure 6. Effect of PHC on protein expression in the lung tissue of rats with LPS-induced acute lung injury. (A) Western blot analysis of Cav-1, TLR4, p38 MAPK, p-p38 MAPKs and nuclear NF-kB p65 protein expression. Relative protein expression levels of (B) Cav-1, (C) TLR4, (D) p-p38 MAPKs (p-p38 MAPKs/p38 MAPKs) and (E) nuclear NF-kB p65. The data are presented as the mean \pm standard error of the mean $(n=10)$. " $\mathrm{P}<0.05$ vs. C group. ${ }^{~} \mathrm{P}<0.05$ vs. LPS group. PHC/P, penehyclidine hydrochloride; LPS, lipopolysaccharide; Cav-1, caveolin-1; TLR4, toll-like receptor 4; p, phosphorylated; p38 MAPKs, p38 mitogen-activated protein kinases; NF-kB p65, nuclear factor kappa-light-chain-enhancer of activated B cells transcription factor p65 subunit; C, control.
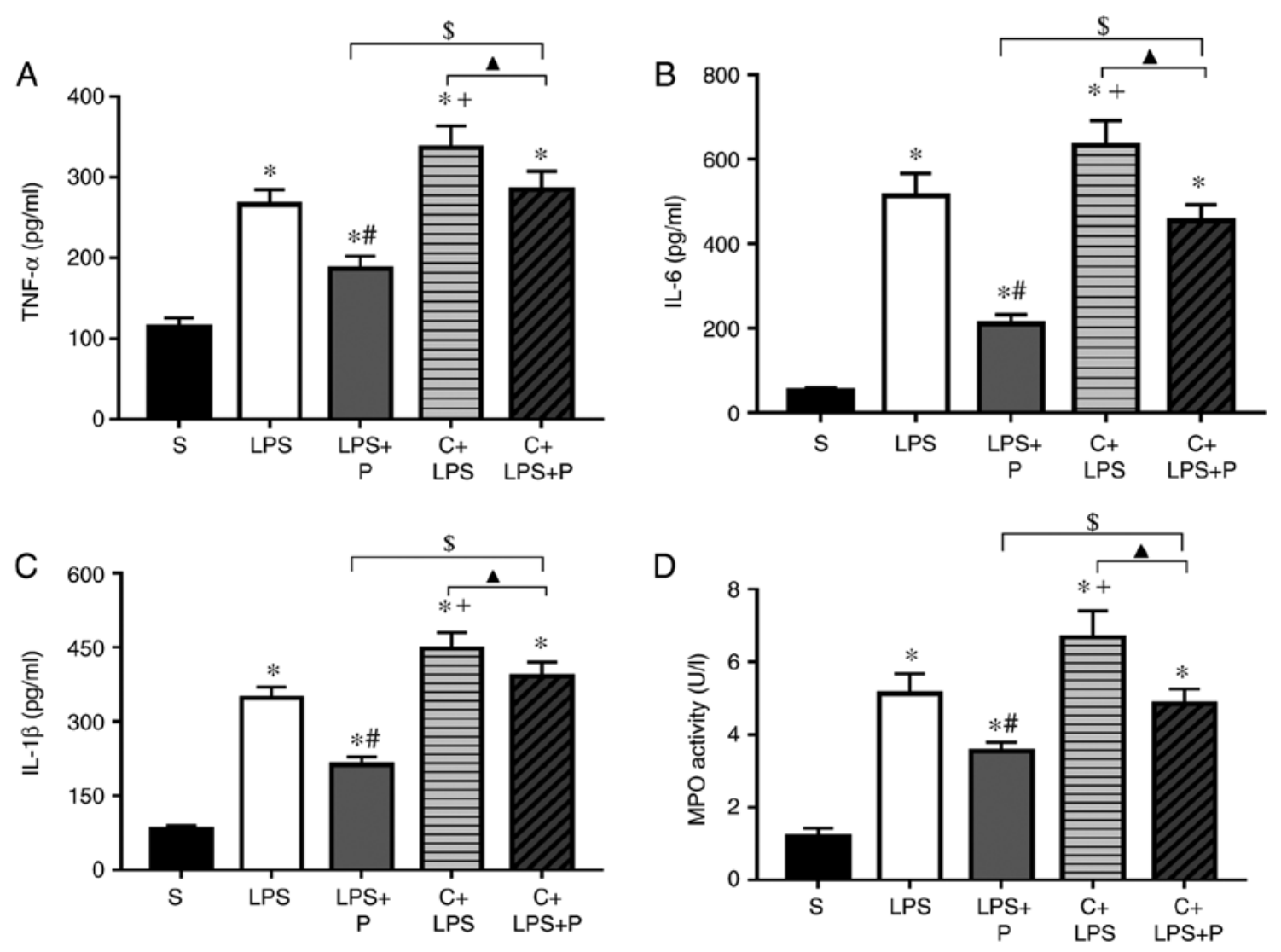

Figure 7. Effect of PHC on pro-inflammatory cytokine production in cell culture supernatant and MPO activity in LPS-stimulated J774A.1 cells. (A) TNF- $\alpha$, (B) IL-6, (C) IL-1 $\beta$ and (D) MPO activity. The data are presented as the mean \pm standard error of the mean ( $\mathrm{n}=5$ ). ${ }^{*} \mathrm{P}<0.05$ vs. $\mathrm{S}$ group. ${ }^{*} \mathrm{P}<0.05$ vs. LPS group. ${ }^{+} \mathrm{P}<0.05$ vs. LPS group. ${ }^{~} \mathrm{P}<0.05$ vs. LPS $+\mathrm{P}$ group. ${ }^{\star} \mathrm{P}<0.05$ vs. $\mathrm{C}+$ LPS group. MPO, myeloperoxidase; TNF- $\alpha$, tumor necrosis factor- $\alpha$; IL, interleukin; PHC/P, penehyclidine hydrochloride; LPS, lipopolysaccharide; C, Cav-1-siRNA; S, Scramble-siRNA.

Wang et al (19) revealed that Cav-1 regulated LPS induced pro-inflammatory cytokines TNF- $\alpha$ and IL- 6 production in murine peritoneal and alveolar macrophages via the p38 MAPKs pathway. In the present study, the results indicated 



Figure 8. Effect of PHC on protein expression levels in LPS-stimulated J774A.1 cells. (A) Western blot analysis of Cav-1 and relative protein expression levels of Cav-1 in J774A.1 cells transfected with Scr-siRNA or Cav-1-siRNA. Data are presented as the mean \pm standard error of the mean $(n=5)$. $P<0.05$ vs. Scr-siRNA group. (B) Western blot analysis of Cav-1, TLR4, p38 MAPK, p-p38 MAPKs and nuclear NF- $\mathrm{kB}$ p65 protein expression. Relative protein expression levels of (C) Cav-1, (D) TLR4, (E) p-p38 MAPKs (p-p38 MAPKs/p38 MAPKs) and (F) nuclear NF-kB p65. Data are presented as the mean \pm standard error of the mean $(\mathrm{n}=5)$. ${ }^{.} \mathrm{P}<0.05$ vs. $\mathrm{S}$ group. ${ }^{\#} \mathrm{P}<0.05$ vs. LPS group. ${ }^{+} \mathrm{P}<0.05$ vs. $\mathrm{LPS}$ group. ${ }^{\mathrm{P}} \mathrm{P}<0.05$ vs. $\mathrm{LPS}+\mathrm{P}$ group. ${ }^{\wedge} \mathrm{P}<0.05$ vs. $\mathrm{C}+\mathrm{LPS}$ group. $\mathrm{PHC} / \mathrm{P}$, penehyclidine hydrochloride; LPS, lipopolysaccharide; Cav-1, caveolin-1; TLR4, Toll-like receptor 4; NF-кB p65, nuclear factor kappa-light-chain-enhancer of activated B cells transcription factor 65 subunit; p, phosphorylated; p38 MAPKs, p38 mitogen-activated protein kinases; C, Cav-1-siRNA; siRNA, short interfering RNA; S, scramble-siRNA.

that Cav-1 knockdown by siRNA in J774A.1 macrophages increased pro-inflammatory cytokine production and MPO activity induced by LPS, concomitant with the activation of the TLR4/NF- $\kappa$ B and p38 MAPKs pathways. It was also indicated that PHC upregulated Cav-1 expression following LPS stimulation in vivo and in vitro.

There are a number of limitations in the present study. Whether LPS-induced lung injury is aggravated in Cav-1 gene deficiency requires additional investigation through the use of Cav-1 gene-deficient mice. Whether other pathways have the ability to mediate Cav-1 involvement in lung injury following LPS-induced ALI also requires additional studies. Furthermore, a single macrophage cell line was selected in the present study; lung epithelial cells or other alveolar macrophage cell lines should be included in future studies.

In conclusion, the present study indicated that PHC possesses anti-inflammatory activity in rats with ALI and
LPS-stimulated J774A.1 cells, which may due to the inhibition of p38 MAPKs phosphorylation and TLR4/NF- $\mathrm{BB}$ signaling pathway by upregulating Cav-1 expression.

\section{Acknowledgements}

Not applicable.

\section{Funding}

The present study was supported by the National Natural Science Foundation of China (grant no. 81571941).

\section{Availability of data and materials}

The datasets used or analyzed during the current study are available from the corresponding author on reasonable request. 


\section{Authors' contributions}

QK and XW contributed equally to the present study. XW, ZX and XS conceived and designed the study. QK, XW, LZ and WD performed the experiments, analyzed the data, interpreted the experimental results, prepared the figures and drafted the manuscript. ZX, LZ and XS were involved in revising the manuscript. All authors read and approved the final version of the manuscript.

\section{Ethics approval and consent to participate}

Ethical approval was provided by the Medical Ethics Committee of Renmin Hospital of Wuhan University. All surgical procedures were performed in accordance with Wuhan University Animal Care and Use Committee.

\section{Patient consent for publication}

Not applicable.

\section{Competing interests}

The authors declare that they have no competing interests.

\section{References}

1. Strauss RH, Palmer KC and Hayes JA: Acute lung injury induced by cadmium aerosol. I. Evolution of alveolar cell damage. Am J Pathol 84: 561-578, 1976.

2. Ware LB and Matthay MA: The acute respiratory distress syndrome. N Engl J Med 342: 1334-1349, 2000.

3. Fein AM and Calalang-Colucci MG: Acute lung injury and acute respiratory distress syndrome in sepsis and septic shock. Crit Care Clin 16: 289-317, 2000.

4. Bhatia M and Moochhala S: Role of inflammatory mediators in the pathophysiology of acute respiratory distress syndrome. J Pathol 202: 145-156, 2004.

5. Rubenfeld GD, Caldwell E, Peabody E, Weaver J, Martin DP, Neff M, Stern EJ and Hudson LD: Incidence and outcomes of acute lung injury. N Engl J Med 353: 1685-1693, 2005.

6. Matute-Bello G, Frevert CW and Martin TR: Animal models of acute lung injury. Am J Physiol Lung Cell Mol Physiol 295 : L379-L399, 2008.

7. Tolle LB and Standiford TJ: Danger-associated molecular patterns (DAMPs) in acute lung injury. J Pathol 229: 145-156, 2013.

8. Ghosh S, May MJ and Kopp EB: NF-kappa B and Rel proteins: Evolutionarily conserved mediators of immune responses. Annu Rev Immunol 16: 225-260, 1998.

9. Kolb M, Margetts PJ, Anthony DC, Pitossi F and Gauldie J: Transient expression of IL-1beta induces acute lung injury and chronic repair leading to pulmonary fibrosis. J Clin Invest 107: 1529- 1536, 2001

10. Haddad EB, Birrell M, McCluskie K, Ling A, Webber SE, Foster ML and Belvisi MG: Role of p38 MAP kinase in LPS-induced airway inflammation in the rat. Br J Pharmacol 132: $1715-1724,2001$.

11. Nash SP and Heuertz RM: Blockade of p38 map kinase inhibits complement- induced acute lung injury in a murine model. Int Immunopharmacol 5: 1870-1880, 2005.

12. Kim HJ, Lee HS, Chong YH and Kang JL: p38 Mitogen-activated protein kinase up-regulates LPS-induced NF-kappaB activation in the development of lung injury and RAW 264.7 macrophages. Toxicology 225: 36-47, 2006.

13. Han XY, Liu H, Liu CH, Wu B, Chen LF, Zhong BH and Liu KL: Synthesis of the optical isomers of a new anticholinergic drug, penehyclidine hydrochloride (8018). Bioorg Med Chem Lett 15: 1979-1982, 2005.

14. Shen W, Gan J, Xu S, Jiang G and Wu H: Penehyclidine hydrochloride attenuates LPS-induced acute lung injury involvement of NF-kappaB pathway. Pharmacol Res 60: 296-302, 2009.
15. Benlimame N, Le PU and Nabi IR: Localization of autocrine motility factor receptor to caveolae and clathrin-independent internalization of its ligand to smooth endoplasmic reticulum. Mol Biol Cell 9: 1773-1786, 1998.

16. Gumbleton M, Abulrob AG and Campbell L: Caveolae: An alternative membrane transport compartment. Pharm Res 17: 1035-1048, 2000.

17. Rothberg KG, Heuser JE, Donzell WC, Ying YS, Glenney JR and Anderson RG: Caveolin, a protein component of caveolae membrane coats. Cell 68: 673-682, 1992.

18. Sun Y, Hu G, Zhang X and Minshall RD: Phosphorylation of caveolin-1 regulates oxidant-induced pulmonary vascular permeability via paracellular and transcellular pathways. Circ Res 105: 676-685, 15 p following 685, 2009.

19. Wang XM, Kim HP, Song R and Choi AM: Caveolin-1 confers antiinflammatory effects in murine macrophages via the MKK3/p38 MAPK pathway. Am J Respir Cell Mol Biol 34: 434-442, 2006.

20. Garrean S, Gao XP, Brovkovych V, Shimizu J, Zhao YY, Vogel SM and Malik AB: Caveolin-1 regulates NF-kappaB activation and lung inflammatory response to sepsis induced by lipopolysaccharide. J Immunol 177: 4853-4860, 2006.

21. National Research Council (US) Committee on Recognition and Alleviation of Pain in Laboratory Animals: Recognition and Alleviation of Pain in Laboratory Animals. National Academies Press, Washington, DC, 2009.

22. Wu XJ, Xia ZY, Wang LL, Luo T, Zhan LY, Meng QT and Song XM: Effects of penehyclidine hydrochloride on pulmonary contusion from blunt chest trauma in rats. Injury 43: 232-236, 2012.

23. Wu XJ, Liu HM, Song XM, Zhao B, Leng Y, Wang EY, Zhan LY, Meng QT and Xia ZY: Penehyclidine hydrochloride inhibits TLR4 signaling and inflammation, and attenuates blunt chest trauma and hemorrhagic shock-induced acute lung injury in rats. Mol Med Rep 17: 6327-6336, 2018.

24. Su X, Wang L, Song Y and Bai C: Inhibition of inflammatory responses by ambroxol, a mucolytic agent, in a murine model of acute lung injury induced by lipopolysaccharide. Intensive Care Med 30: 133-140, 2004.

25. Matthay MA, McAuley DF and Ware LB: Clinical trials in acute respiratory distress syndrome: Challenges and opportunities. Lancet Respir Med 5: 524-534, 2017.

26. Brigham KL and Meyrick B: Endotoxin and lung injury. Am Rev Respir Dis 133: 913-927, 1986.

27. Kitamura Y, Hashimoto S, Mizuta N, Kobayashi A, Kooguchi K, Fujiwara I and Nakajima H: Fas/FasL-dependent apoptosis of alveolar cells after lipopolysaccharide- induced lung injury in mice. Am J Respir Crit Care Med 163: 762-769, 2001.

28. Chiang EY, Yu X and Grogan JL: Immune complex-mediated cell activation from systemic lupus erythematosus and rheumatoid arthritis patients elaborate different requirements for IRAK1/4 kinase activity across human cell types. J Immunol 186: 1279-1288, 2011.

29. Kagan JC and Medzhitov R: Phosphoinositide-mediated adaptor recruitment controls toll-like receptor signaling. Cell 125: 943-955, 2006.

30. Wilson SJ, Leone BA, Anderson D, Manning A and Holgate ST: Immunohistochemical analysis of the activation of NF-kappaB and expression of associated cytokines and adhesion molecules in human models of allergic inflammation. J Pathol 189: 265-272, 1999.

31. Karin M and Ben-Neriah Y: Phosphorylation meets ubiquitination: The control of NF-[kappa]B activity. Annu Rev Immunol 18: 621-663, 2000.

32. Kuo MY, Liao MF, Chen FL, Li YC, Yang ML, Lin RH and Kuan YH: Luteolin attenuates the pulmonary inflammatory response involves abilities of antioxidation and inhibition of MAPK and NFкB pathways in mice with endotoxin-induced acute lung injury. Food Chem Toxicol 49: 2660-2666, 2011.

33. Yeh CH, Yang JJ, Yang ML, Li YC and Kuan YH: Rutin decreases lipopolysaccharide-induced acute lung injury via inhibition of oxidative stress and the MAPK-NF- $\kappa B$ pathway. Free Radic Biol Med 69: 249-257, 2014

34. Yang J, Murphy C, Denham W, Botchkina G, Tracey KJ and Norman J: Evidence of a central role for p38 map kinase induction of tumor necrosis factor alpha in pancreatitis-associated pulmonary injury. Surgery 126: 216-222, 1999.

35. Lee JC and Young PR: Role of CSB/p38/RK stress response kinase in LPS and cytokine signaling mechanisms. J Leukoc Biol 59: 152-157, 1996. 
36. Carter AB, Knudtson KL, Monick MM and Hunninghake GW: The p38 mitogen-activated protein kinase is required for NF-kappaB-dependent gene expression. The role of TATA-binding protein (TBP). J Biol Chem 274: 30858-30863, 1999.

37. Schnyder-Candrian S, Quesniaux VF, Di Padova F, Maillet I, Noulin N, Couillin I, Moser R, Erard F, Vargaftig BB, Ryffel B and Schnyder B: Dual effects of p38 MAPK on TNF-dependent bronchoconstriction and TNF-independent neutrophil recruitment in lipopolysaccharide-induced acute respiratory distress syndrome. J Immunol 175: 262-269, 2005.

38. Zhan J, Wang Y, Wang C, Li J, Zhang Z and Jia B: Protective effects of penehyclidine hydrochloride on septic mice and its mechanism. Shock 28: 727-732, 2007.
39. Yuan K, Huang C, Fox J, Gaid M, Weaver A, Li G, Singh BB, Gao $\mathrm{H}$ and $\mathrm{Wu} \mathrm{M}$ : Elevated inflammatory response in caveolin-1-deficient mice with Pseudomonas aeruginosa infection is mediated by STAT3 protein and nuclear factor kappaB (NF-kappaB). J Biol Chem 286: 21814-21825, 2011.

(i) (9) This work is licensed under a Creative Common Attribution-NonCommercial-NoDerivatives 4.0 International (CC BY-NC-ND 4.0) License. 